\title{
How are Journal Impact, Prestige and Article Influence Related? An Application to Neuroscience*
}

\author{
Chia-Lin Chang \\ Department of Applied Economics \\ Department of Finance \\ National Chung Hsing University \\ Taichung, Taiwan \\ Michael McAleer \\ Econometric Institute \\ Erasmus School of Economics \\ Erasmus University Rotterdam \\ and \\ Tinbergen Institute \\ The Netherlands \\ and \\ Department of Quantitative Economics \\ Complutense University of Madrid \\ Spain

\section{Les Oxley} \\ Department of Economics and Finance \\ University of Canterbury \\ New Zealand
}

Revised: June 2011

* The authors wish to thank three referees for helpful comments and suggestions. For financial support, the first author acknowledges the National Science Council, Taiwan; the second author acknowledges the Australian Research Council, National Science Council, Taiwan, and the Japan Society for the Promotion of Science; and the third author acknowledges the Royal Society of New Zealand, Marsden Fund. 


\begin{abstract}
The paper analyses the leading journals in Neurosciences using quantifiable Research Assessment Measures (RAM), highlights the similarities and differences in alternative RAM, shows that several RAM capture similar performance characteristics of highly cited journals, and shows that some other RAM have low correlations with each other, and hence add significant informational value. Alternative RAM are discussed for the Thomson Reuters ISI Web of Science database (hereafter ISI). The RAM that are calculated annually or updated daily include the classic 2-year impact factor (2YIF), 5-year impact factor (5YIF), Immediacy (or zero-year impact factor (OYIF)), Eigenfactor score, Article Influence score, C3PO (Citation Performance Per Paper Online), h-index, Zinfluence, PI-BETA (Papers Ignored By Even The Authors), 2-year and historical Self-citation Threshold Approval Ratings (STAR), Impact Factor Inflation (IFI), and Cited Article Influence (CAI). The RAM are analysed for 26 highly cited journals in the ISI category of Neurosciences. The paper finds that the Eigenfactor score and PI-BETA are not highly correlated with the other RAM scores, so that they convey additional information regarding journal rankings, that Article Influence is highly correlated with some existing RAM, so that it has little informative incremental value, and that CAI has additional informational value to that of Article Influence. Harmonic mean rankings of the 13 RAM criteria for the 26 highly cited journals are also presented. Emphasizing the 2-year impact factor of a journal to the exclusion of other informative RAM criteria is shown to lead to a distorted evaluation of journal performance and influence, especially given the informative value of several other RAM.
\end{abstract}

Keywords: Impact factor, Prestige, Immediacy, Eigenfactor, Article Influence, h-index, C3PO, Zinfluence, PI-BETA, STAR, IFI, Cited Article influence. 


\section{Introduction}

Encouraging, monitoring and publishing high quality research are fundamental to science, and research assessment rankings are essential to evaluate the research performance of individuals and the quality of academic journals. The perceived research performance of individual researchers can be crucial for hiring, firing, tenure and promotion decisions. In the absence of suitable information regarding the perceived quality of research output, the quality of a journal has frequently been used as a proxy for the research quality of an academic paper.

The perceived quality of a journal is an inappropriate and misleading proxy for the perceived quality of a published paper. The quality, popularity and prestige of a journal are based on outstanding papers that it has previously published. However, a prestigious journal cannot be an accurate reflection of the quality of a recently published paper, especially when the paper has yet received few, if any, citations. Furthermore, Seglen (1997) finds that the citations rates of papers determine the impact factor of journals, but not the reverse.

The acceptance of a paper for publication in a journal is based on the expertise of a subset of the Editor, Co-editor, Associate Editor, and referees, who determine the rejection rate before publication. Experts can, and do, make mistakes. After a paper has been published, the rejection rate of a journal depends on the worldwide academic profession. Consequently, the proportion of published papers that is ignored by the profession, and by even the authors, is an important non-citations performance measure. Researchers worldwide are less likely to make errors regarding the quality of academic research papers that have been published than a small group of editorial experts who are required to make judgments regarding the quality of a paper before publication.

Virtually all RAM are based on citations, which capture impact, popularity, prestige and influence. The Thomson Reuters ISI Web of Science database (hereafter ISI) is a leading high quality database for generating RAM to evaluate the research performance of individual researchers and the quality of academic journals (see Seglen (1997), among others, for caveats regarding ISI data). This paper examines the importance of RAM as viable rankings criteria, highlights the usefulness of existing RAM from ISI, and evaluates the usefulness of 
three new RAM criteria using ISI data for the 26 most highly cited journals in the Neurosciences.

The plan of the remainder of the paper is as follows. Section 2 presents some key RAM using ISI data that are calculated annually or are updated daily, namely the classic 2-year impact factor (2YIF), 5-year impact factor (5YIF), Immediacy (or zero-year impact factor (0YIF)), Eigenfactor score, Article Influence score, C3PO (Citation Performance Per Paper Online), hindex, Zinfluence, PI-BETA (Papers Ignored - By Even The Authors), 2-year and historical Self-citation Threshold Approval Ratings (STAR), Impact Factor Inflation (IFI), and Cited Article Influence (CAI). Section 3 discusses and analyses RAM for the ISI discipline of Neurosciences. Section 4 summarizes the outcomes.

\section{Research Assessment Measures (RAM)}

The RAM are intended as descriptive statistics, and are not based on a mathematical model. Hence, in what follows, no optimization or estimation is required.

\subsection{Annual RAM}

With two exceptions, namely the Eigenfactor and Article Influence scores, existing RAM is reported separately for the sciences and social sciences. RAM may be computed annually or updated daily. The annual RAM given below are calculated for a Journal Citations Reports (JCR) calendar year, which is the year before the annual RAM are released.

\section{(1) 2-year impact factor (2YIF):}

The classic 2-year impact factor (2YIF) of an ISI journal is typically referred to as "the impact factor", is calculated annually, and is defined as "Total citations in a year to papers published in a journal in the previous 2 years / Total papers published in a journal in the previous 2 years”. The choice of 2 years by ISI is arbitrary.

\section{(2) 2-year impact factor without self citations (2YIF*):}


ISI also reports a 2-year impact factor without journal self citations (that is, citations to a journal in which a citing paper is published). As this impact factor is not widely used, we will refer to it as $2 \mathrm{YIF}^{*}$.

\section{(3) 5-year impact factor (5YIF):}

The 5-year impact factor (5YIF) of an ISI journal is calculated annually, and is defined as "Total citations in a year to papers published in a journal in the previous 5 years / Total papers published in a journal in the previous 5 years." The choice of 5 years by ISI is arbitrary.

\section{(4) Immediacy:}

Immediacy is a zero-year impact factor (OYIF) of an ISI journal, is calculated annually, and is defined as "Total citations to papers published in a journal in the same year / Total papers published in a journal in the same year.” The choice of the same year by ISI is arbitrary.

\section{(5) Eigenfactor score:}

The Eigenfactor score (Bergstrom (2007), Bergstrom, West and Wiseman (2008)) is a modified 5YIF, and is calculated annually to capture the prestige of a journal. The Eigenfactor algorithm (see www.eigenfactor.org/methods.htm) effectively ranks journals according to citations and the length of time that researchers are logged on to a journal's website. The Eigenfactor does not check how much time researchers spend reading hard copies of journals.

\section{(6) Article Influence:}

Article Influence measures the relative importance of an ISI journal on a per-article basis, is a standardized Eigenfactor score, and is calculated annually. Article Influence is defined as "Eigenfactor score divided by the fraction of all articles published by a journal."

\section{(7) IFI:}

The ratio of 2YIF to 2YIF* is intended to capture how journal self citations inflate the impact factor of a journal. Impact Factor Inflation (IFI) is defined as "IFI = 2YIF / 2YIF*". The minimum value for IFI is 1 , with any value above the minimum capturing the effect of journal self citations on the 2-year impact factor. 


\section{(8) STAR:}

ISI has implicitly recognized the inflation in journal self citations by calculating an impact factor that excludes self citations, and provides data on journal self citations, both historically and for the preceding two years, in calculating 2YIF. The Self-citation Threshold Approval Rating (STAR) is the difference between citations in other journals and journal self citations. If $S=$ journal self citations, STAR is defined as "STAR $=[(100-S)-S]=(100-2 S)$ ". If $S=0$, 25, 50 or 100, for example, STAR $=100,50,0$ and -100 , respectively. As STAR can be calculated using journal self citations, both historically and for the preceding two years, historical STAR is H-STAR and a 2-year STAR is 2Y-STAR.

\subsection{Daily Updated RAM}

Other RAM are updated daily, and are reported for a given day in a calendar year rather than for a JCR year.

\section{(9) C3PO:}

ISI reports the mean number of citations for an ISI journal, namely total citations up to a given day divided by the number of papers published in an ISI journal up to the same day, as the "average" number of citations. In order to distinguish the mean from the median and mode, the C3PO of an ISI journal on any given day is defined by Chang et al. (2011) as “C3PO (Citation Performance Per Paper Online) = Total citations to a journal / Total papers published in a journal." [Note: C3PO should not be confused with C-3PO, the Star Wars android.]

\section{(10) h-index:}

The h-index (Hirsch, 2005)) was originally proposed to assess the scientific research productivity and citations impact of individual researchers. The h-index can also be calculated for journals, and should be interpreted as assessing the impact or influence of highly cited publications in ISI journals. The h-index of an ISI journal on any given day is based on cited and citing papers, including self citations of ISI journals, and is defined as "hindex = each of $\mathrm{h}$ published papers has been cited at least $\mathrm{h}$ times.”

\section{(11) PI-BETA:}


This RAM measures the proportion of papers in a journal that has never been cited, which is, in effect, a rejection rate after publication. Chang et al. (2011) argue that lack of citations of a published paper, especially if it is not a recent publication, may reflect on the quality of a journal by exposing: (i) what might be considered as incorrect decisions by the editorial board of a journal; and (ii) the lost opportunities of papers that might have been cited had they not been rejected by the journal. Chang et al. (2011) propose that a paper with zero citations in ISI journals can be measured by PI-BETA (= Papers Ignored (PI) - By Even The Authors (BETA)), which is calculated for an ISI journal on any given day as "Number of papers with zero citations in a journal / Total papers published in a journal.”

\section{(12) CAI:}

Article Influence is intended to measure the average influence of an article across the sciences and social sciences. As an article with zero citations cannot have influence, a more suitable measure of the influence of cited articles is Cited Article Influence (CAI), which is defined as “CAI = $(1-$ PI-BETA $)($ Article Influence)". If PI-BETA $=0$, then CAI is equivalent to Article Influence; if PI-BETA $=1$, then CAI $=0$. As Article Influence is calculated annually, whereas PI-BETA is updated daily, CAI may be updated daily.

\section{Analysis of ISI RAM Data for Neurosciences}

Bergstrom et al. (2008) analysed “Article Influence Scores and total articles published for the top 25 journals by Eigenfactor score in the field of Neurosciences” (Figure 1, p. 11434). Seven leading journals are highlighted in the figure. Six of these journals are in the ISI category of Neurosciences, with 221 journals, while the seventh, Neurology, is in the ISI category of Clinical Neurology, with 156 journals, where it is ranked fourth according to 2YIF.

In Table 1 we evaluate the 25 most highly cited journals, according to 2YIF, in the Neurosciences, as well as in the Neurology journal. Only articles from ISI Web of Science are included in the citation data. Data for all journals were downloaded from ISI on 12 June 2010 for all citations for 1988-2010, so that citations were counted from 1988 for all papers published in an ISI journal since 1988. As ISI does not provide daily updates for more than 
10,000 articles for purposes of calculating the h-index, C3PO, PI-BETA and CAI, the initial years of several journals were chosen so that no journal had more than 10,000 articles. Owing to the large numbers of articles published in some journals, data for the following four journals started after 1988, namely Annals of Neurology (from 1998), Biological Psychiatry (from 2001), Journal of Neuroscience (from 2004), and Neurology (from 2008).

As can be seen from Table 1, the means and ranges of 2YIF are, respectively, 10.01 and (5.694, 26.405), of 2YIF* are 9.567 and (4.682, 26.190), of 5YIF are 11.291 and (5.516, 31.209), and of Immediacy are 1.984 and $(0.535,4.859)$. As journal self citations in the neurosciences seems relatively low, with a mean IFI of 1.056, the range of IFI is (1.011, 1.216), with the second highest IFI score being 1.101. The h-index has a mean of 134.923 and a wide range of $(10,325)$, and C3PO has a mean of 41.825 and a wide range of $(0.99$, 235.60). The Eigenfactor has a mean of 0.091 and a range of $(0.00433,0.52179)$, with Journal of Neuroscience having the highest score. H-STAR and 2Y-STAR are reasonably high, with only one journal having a score below 70, which accords with a journal self citation rate of $15 \%$.

The PI-BETA outcomes are revealing. The mean is 0.3 , so that, on average, $30 \%$ of papers published in the leading 26 journals in neurosciences are not cited. Only 6 journals have less than $10 \%$ of papers that have never been cited, and 7 journals have PI-BETA in the range $(10,19)$. Annual Review of Neuroscience has an extraordinarily low PI-BETA score of 0.0002. Beyond the $20 \%$ mark, 4 journals have $20-29 \%$ of papers that have never been cited, one journal has $43.91 \%$ of papers that have never been cited, 3 journals are in the range 50 $59 \%$, 4 journals are in the range $60-69 \%$, and one journal has $83.31 \%$ of papers that have never been cited. It should be emphasized that these are the leading journals in the neurosciences, which is revealing.

Article Influence has a mean of 4.947 and a range of $(1.718,18.915)$. As CAI is Article Influence multiplied by (1 - PI-BETA), it is not surprising that Article Influence is different from CAI, which has a mean of 3.643 and a wider range than Article Influence of (0.349, 18.876).

The simple correlations of the 13 RAM for the 26 highly cited journals in the neurosciences are given in Table 2. The 7 pairs of RAM for which the correlations exceed 0.95 (in absolute 
value) are (2YIF, 2YIF*), (2YIF, 5YIF), (2YIF*, 5YIF), (2YIF, Article Influence), (2YIF*, Article Influence), (5YIF, Article Influence), and (IFI, 2Y-STAR). Three RAM pairs for which the simple correlations are in the range $(0.90,0.95)$ are (C3PO, CAI), (CAI, Article Influence), and (H-STAR, 2Y-STAR).

Fersht (2009) showed that there was a very strong positive correlation between the Eigenfactor score and the total number of journal citations, with a correlation coefficient of 0.968 for the top 200 cited ISI journals in 2007. Such a high correlation is not entirely surprising as it captures the size effect of journals, with the total number of publications and total citations typically being positively and highly correlated.

It is not surprising that Article Influence and 5YIF are very highly and positively correlated (at 0.98 ) as the former is a modification of the latter. Given the very high correlation between 5YIF and Article Influence, the modification of 5YIF would seem to be unnecessary, at least for neurosciences.

The Eigenfactor and PI-BETA scores are the only two RAM that have very low simple correlations with each of the other RAM. Therefore, theses two RAM convey useful additional information to what is contained in the other RAM.

It remains to be seen whether an emphasis on the 2-year impact factor of a journal, to the exclusion of other informative RAM, can lead to a distorted evaluation of journal performance and influence. In order to summarize the 13 RAM criteria, 9 of which, namely 2YIF, 2YIF*, 5YIF, Immediacy, IFI, C3PO, PI-BETA, Article Influence and CAI, are based on ratios, the rankings of the 26 journals in neurosciences given in Table 3 are based on the harmonic mean. The rankings of the 26 journals in Table 3 were also obtained using the geometric and arithmetic means, with ranking correlations given as follows:

(i) correlation (harmonic mean, geometric mean) $=0.679$;

(ii) correlation (harmonic mean, arithmetic mean) $=0.702$;

(iii) correlation (geometric mean, arithmetic mean) $=0.845$.

Only the first three journals, namely Annual Review of Neuroscience, Nature Reviews Neuroscience and Neuron, and the number 15 ranked journal, Neuroscience and 
Biobehavioral Reviews, remain unchanged. Four journals to have moved up considerably are Current Opinion in Neurobiology (from 14 to 6), Journal of Neuroscience (from 16 to 8), Brain Research Reviews (from 19 to 10), and Neuroscientist (from 24 to 14). Journals to have moved appreciably in the opposite direction are Behavioral and Brain Sciences (from 5 to 11), Annals of Neurology (from 9 to 16), and Biological Psychiatry (from 13 to 19). All other journals have moved by 5 or fewer positions in either direction.

It is clear that the harmonic mean penalizes uneven rankings across the 13 RAM. Equivalently, the harmonic mean rewards journals with at least one very strong performance, leading to a high ranking.

A linear regression, with Article Influence as a function of 5YIF, is shown in Figure 1 to relate journal impact and article influence. The estimated model shows that Article Influence increases, on average, by 0.6266 for each unit increase in 5YIF. The goodness-of-fit measure, as given by R-squared $=0.9605$, shows that Article Influence can be estimated reasonably accurately on the basis of a linear relationship between Article Influence and 5YIF. As 5YIF is already accessible in ISI, it would seem to be easier and less time consuming to estimate Article Influence using a regression model than using the algorithm to calculate the Eigenfactor score and Article Influence developed by Eigenfactor ${ }^{\mathrm{TM}}$ metrics.

Figure 2 shows a linear regression of 2 YIF as a function of 5YIF to relate the two journal impact factors. As 5YIF includes the citations in 2YIF, the estimated model shows that 2YIF increases, on average, by 0.8293 for each unit increase in 5YIF. The goodness-of-fit measure, as given by R-squared $=0.9443$, shows that 2 YIF can be estimated reasonably accurately on the basis of a linear relationship with 5YIF, and vice-versa.

Figure 3 shows a linear regression, with Article Influence as a function of 2YIF, to relate journal impact and article influence. The estimated model shows that Article Influence increases, on average, by 0.7211 for each unit increase in 2YIF. The goodness-of-fit measure, as given by R-squared $=0.9265$, shows that Article Influence can be estimated reasonably accurately on the basis of a linear relationship between Article Influence and 2YIF, though not as accurately as relating Article Influence to 5YIF in Figure 1. 
A linear regression of Cited Article influence (CAI) as a function of Article Influence is given in Figure 4. The estimated model shows that CAI increases, on average, by 0.835 for each unit increase in Article Influence. The goodness-of-fit measure, as given by R-squared = 0.825, shows that CAI cannot be estimated accurately on the basis of a linear relationship with Article Influence, so that CAI would seem to have additional informational value to that contained in Article Influence.

\section{Conclusion}

The paper analysed the leading journals in Neurosciences using quantifiable Research Assessment Measures (RAM). Alternative RAM criteria were discussed for the Thomson Reuters ISI Web of Science database (hereafter ISI). The RAM criteria were analysed for 26 highly cited journals in the ISI category of Neurosciences. The paper highlighted the similarities and differences in alternative RAM, and showed that several RAM were highly correlated with existing RAM, so that they had little informative incremental value in capturing the performance characteristics of highly cited journals. The paper also showed that the Eigenfactor score and PI-BETA were not highly correlated with the other RAM scores, so that they conveyed additional information regarding journal rankings. Moreover, Cited Article Influence (CAI) was shown to have additional informational value to Article Influence.

Harmonic mean rankings of the 13 RAM were also presented for the 26 highly cited journals in neurosciences. It was shown that emphasizing the 2-year impact factor of a journal to the exclusion of other informative RAM could lead to a distorted evaluation of journal impact, prestige, performance and influence. Therefore, the harmonic mean rankings give a more robust journal ranking than relying solely on the 2-year impact factor. 


\section{References}

Bergstrom C. (2007), Eigenfactor: Measuring the value and prestige of scholarly journals, C\&RL News, 68, 314-316.

Bergstrom, C.T., J.D. West and M.A. Wiseman (2008), The Eigenfactor ${ }^{\mathrm{TM}}$ metrics, Journal of Neuroscience, 28(45), 11433-11434 (November 5, 2008).

Chang, C.-L., M. McAleer and L. Oxley (2011), Great expectatrics: Great papers, great journals, great econometrics. Econometric Reviews, 30(6), 583-619.

Fersht, A. (2009), The most influential journals: Impact factor and Eigenfactor, Proceedings of the National Academy of Sciences of the United States of America, 106(17), 6883-6884 (April 28, 2009).

ISI Web of Science (2010), Journal Citation Reports, Essential Science Indicators, Thomson Reuters ISI.

Seglen, P.O. (1997), Why the impact factor of journals should not be used for evaluating research, BMJ: British Medical Journal, 314(7079), 498-502. 
Table 1

Research Assessment Measures (RAM) for 26 Neuroscience Journals

\begin{tabular}{|c|c|c|c|c|c|c|c|c|c|c|c|c|c|}
\hline Journal & 2YIF & 2YIF* & $5 \mathrm{YIF}$ & IFI & Immediacy & h-index & $\mathrm{C} 3 \mathrm{PO}$ & PI-BETA & Eigenfactor & CAI & $\begin{array}{c}\text { Article } \\
\text { Influence }\end{array}$ & $\begin{array}{c}\mathrm{H}- \\
\text { STAR }\end{array}$ & $\begin{array}{c}\text { 2Y- } \\
\text { STAR }\end{array}$ \\
\hline Annual Review of Neuroscience & 26.405 & 26.190 & 31.209 & 1.008 & 3.348 & 183 & 235.60 & 0.0020 & 0.04611 & 18.876 & 18.915 & 100 & 100 \\
\hline Nature Reviews Neuroscience & 25.497 & 24.940 & 26.678 & 1.022 & 4.859 & 169 & 42.50 & 0.5749 & 0.11399 & 5.925 & 13.939 & 98 & 98 \\
\hline Neuron & 14.170 & 13.370 & 14.857 & 1.060 & 2.599 & 325 & 96.78 & 0.0696 & 0.28702 & 7.719 & 8.296 & 92 & 90 \\
\hline Nature Neuroscience & 14.164 & 13.841 & 16.825 & 1.023 & 3.297 & 201 & 56.00 & 0.1572 & 0.19666 & 7.687 & 9.120 & 98 & 96 \\
\hline Behavorial and Brain Sciences & 12.818 & 11.318 & 19.355 & 1.133 & 2.667 & 109 & 5.22 & 0.5654 & 0.01173 & 3.709 & 8.533 & 92 & 78 \\
\hline Trends in Neurosciences & 12.817 & 12.640 & 14.475 & 1.014 & 1.925 & 235 & 80.30 & 0.1048 & 0.06325 & 6.186 & 6.910 & 100 & 98 \\
\hline Molecular Psychiatry & 12.537 & 11.932 & 11.937 & 1.051 & 4.161 & 102 & 25.59 & 0.2111 & 0.04612 & 3.351 & 4.247 & 92 & 92 \\
\hline Trends in Cognitive Sciences & 10.981 & 10.560 & 13.826 & 1.040 & 1.086 & 120 & 33.29 & 0.2669 & 0.05323 & 4.633 & 6.320 & 98 & 94 \\
\hline Annals of Neurology & 9.935 & 9.429 & 9.081 & 1.054 & 2.166 & 145 & 13.79 & 0.6106 & 0.07996 & 1.300 & 3.337 & 96 & 90 \\
\hline Brain & 9.603 & 9.153 & 9.808 & 1.049 & 1.593 & 198 & 53.68 & 0.1106 & 0.09864 & 3.137 & 3.527 & 94 & 92 \\
\hline Progress in Neurobiology & 9.130 & 8.900 & 12.346 & 1.026 & 1.169 & 163 & 78.33 & 0.0563 & 0.02492 & 4.478 & 4.745 & 98 & 96 \\
\hline Frontiers in Neuroendocrinology & 8.692 & 8.385 & 11.288 & 1.037 & 3.188 & 10 & 1.12 & 0.6553 & 0.00433 & 1.142 & 3.314 & 96 & 94 \\
\hline Biological Psychiatry & 8.672 & 8.305 & 9.015 & 1.044 & 1.943 & 114 & 9.29 & 0.6730 & 0.11389 & 1.042 & 3.186 & 94 & 92 \\
\hline Current Opinion in Neurobiology & 8.102 & 7.946 & 9.143 & 1.020 & 0.535 & 136 & 53.65 & 0.0949 & 0.05407 & 4.562 & 5.040 & 100 & 98 \\
\hline Neuroscience and Biobehavioral Reviews & 7.804 & 7.641 & 9.563 & 1.021 & 1.426 & 120 & 41.06 & 0.1016 & 0.02488 & 2.810 & 3.128 & 96 & 96 \\
\hline Journal of Neuroscience & 7.452 & 6.771 & 8.122 & 1.101 & 1.138 & 108 & 19.92 & 0.1429 & 0.52179 & 3.038 & 3.544 & 86 & 82 \\
\hline Neurology (ISI: Clinical Neurology) & 7.043 & 6.675 & 6.857 & 1.055 & 1.796 & 31 & 0.99 & 0.8331 & 0.17775 & 0.349 & 2.089 & 94 & 90 \\
\hline Neuropsychopharmacology & 6.835 & 6.444 & 6.716 & 1.061 & 2.106 & 116 & 16.55 & 0.4391 & 0.05970 & 1.214 & 2.164 & 92 & 90 \\
\hline Brain Research Reviews & 6.236 & 6.062 & 7.260 & 1.029 & 1.972 & 128 & 63.33 & 0.0511 & 0.02113 & 2.400 & 2.529 & 98 & 96 \\
\hline Sleep Medicine Reviews & 6.143 & 5.873 & 6.630 & 1.046 & 1.690 & 43 & 16.33 & 0.1807 & 0.00753 & 1.808 & 2.207 & 92 & 92 \\
\hline Pain & 6.030 & 5.235 & 6.653 & 1.152 & 1.038 & 159 & 34.15 & 0.1296 & 0.05218 & 1.495 & 1.718 & 88 & 84 \\
\hline Neurobiology of Aging & 5.959 & 5.768 & 6.132 & 1.033 & 1.591 & 109 & 9.28 & 0.6039 & 0.03439 & 0.761 & 1.921 & 92 & 94 \\
\hline Cerebal Cortex & 5.907 & 5.515 & 6.939 & 1.071 & 1.619 & 131 & 37.00 & 0.0843 & 0.06793 & 2.864 & 3.128 & 88 & 88 \\
\hline Neuroscientist & 5.896 & 5.833 & 6.455 & 1.011 & 0.667 & 54 & 8.81 & 0.5657 & 0.01391 & 1.127 & 2.594 & 98 & 98 \\
\hline $\begin{array}{l}\text { Journal of Cerebral Blood Flow and } \\
\text { Metabolism }\end{array}$ & 5.741 & 5.340 & 5.516 & 1.075 & 1.027 & 149 & 33.06 & 0.2312 & 0.03189 & 1.435 & 1.866 & 90 & 88 \\
\hline Neuroimage & 5.694 & 4.682 & 6.884 & 1.216 & 0.984 & 150 & 21.83 & 0.2793 & 0.15549 & 1.670 & 2.317 & 68 & 66 \\
\hline Mean & 10.010 & 9.567 & 11.291 & 1.056 & 1.984 & 134.923 & 41.825 & 0.300 & 0.091 & 3.643 & 4.947 & 93.462 & 91.231 \\
\hline
\end{tabular}

Note: Citations data were downloaded from ISI on 12 June 2010 for 1988-2010. 
Table 2

Correlations of 13 RAM Criteria for 26 Neuroscience Journals

\begin{tabular}{|c|c|c|c|c|c|c|c|c|c|c|c|c|c|}
\hline RAM & 2YIF & 2 YIF* $^{*}$ & 5YIF & IFI & Immediacy & h-index & СЗРО & $\begin{array}{c}\text { PI- } \\
\text { BETA }\end{array}$ & Eigenfactor & CAI & $\begin{array}{l}\text { Article } \\
\text { Influen } \\
\text { ce }\end{array}$ & $\begin{array}{c}\text { H- } \\
\text { STAR }\end{array}$ & $\begin{array}{l}\text { 2Y- } \\
\text { STAR }\end{array}$ \\
\hline 2YIF & 1.000 & & & & & & & & & & & & \\
\hline 5YIF & 0.972 & 0.966 & 1.000 & & & & & & & & & & \\
\hline IFI & -0.318 & -0.365 & -0.268 & 1.000 & & & & & & & & & \\
\hline СЗРО & 0.654 & 0.669 & 0.666 & -0.308 & 0.225 & 0.572 & 1.000 & & & & & & \\
\hline PI-BETA & -0.080 & -0.087 & -0.108 & 0.024 & 0.177 & -0.548 & -0.601 & 1.000 & & & & & \\
\hline Eigenfactor & 0.058 & 0.042 & 0.002 & 0.251 & -0.006 & 0.273 & -0.005 & -0.092 & 1.000 & & & & \\
\hline CAI & 0.819 & 0.826 & 0.844 & -0.304 & 0.411 & 0.526 & 0.930 & -0.470 & 0.084 & 1.000 & & & \\
\hline $\begin{array}{l}\text { Article } \\
\text { Influence }\end{array}$ & 0.963 & 0.961 & 0.980 & -0.290 & 0.618 & 0.442 & 0.745 & -0.184 & 0.064 & 0.908 & 1.000 & & \\
\hline
\end{tabular}

Note: Citations data were downloaded from ISI on 12 June 2010 for 1988-2010. 
Table 3

Harmonic Mean Rankings of 13 RAM Criteria for 26 Neuroscience Journals

\section{Journal}

Annual Review of Neuroscience

Nature Reviews Neuroscience

Neuron

Trends in Neurosciences

Nature Neuroscience

Current Opinion in Neurobiology

Progress in Neurobiology

Journal of Neuroscience

Molecular Psychiatry

Brain Research Reviews

Behavorial and Brain Sciences

Trends in Cognitive Sciences

Brain

Neuroscientist

Neuroscience and Biobehavioral Reviews

Annals of Neurology

Frontiers in Neuroendocrinology

Cerebal Cortex

Biological Psychiatry

Neurology (ISI: Clinical Neurology)

Neuroimage

Pain

Neuropsychopharmacology

Sleep Medicine Reviews

Neurobiology of Aging

Journal of Cerebral Blood Flow and

Metabolism

Note: Citations data were downloaded from ISI on 12 June 2010 for 1988-2010.

\begin{tabular}{|c|c|c|c|c|c|c|c|c|c|c|c|c|c|}
\hline 2YIF & 2YIF* & 5YIF & IFI & Immediacy & h-index & СЗРО & PI-BETA & Eigenfactor & CAI & $\begin{array}{c}\text { Article } \\
\text { Influence }\end{array}$ & $\begin{array}{c}\text { H- } \\
\text { STAR }\end{array}$ & $\begin{array}{c}\text { 2Y- } \\
\text { STAR }\end{array}$ & Rank \\
\hline 1 & 1 & 1 & 1 & 3 & 5 & 1 & 1 & 17 & 1 & 1 & 1 & 1 & 1 \\
\hline 2 & 2 & 2 & 6 & 1 & 6 & 9 & 21 & 6 & 5 & 2 & 4 & 4 & 2 \\
\hline 3 & 4 & 5 & 19 & 7 & 1 & 2 & 4 & 2 & 2 & 5 & 17 & 19 & 3 \\
\hline 6 & 5 & 6 & 3 & 12 & 2 & 3 & 8 & 11 & 4 & 6 & 2 & 2 & 4 \\
\hline 4 & 3 & 4 & 7 & 4 & 3 & 6 & 12 & 3 & 3 & 3 & 5 & 6 & 5 \\
\hline 14 & 14 & 13 & 4 & 26 & 12 & 8 & 6 & 13 & 7 & 8 & 3 & 3 & 6 \\
\hline 11 & 11 & 8 & 8 & 19 & 7 & 4 & 3 & 20 & 8 & 9 & 7 & 7 & 7 \\
\hline 16 & 16 & 16 & 23 & 20 & 21 & 17 & 11 & 1 & 12 & 11 & 25 & 24 & 8 \\
\hline 7 & 6 & 9 & 16 & 2 & 22 & 15 & 14 & 16 & 10 & 10 & 18 & 15 & 9 \\
\hline 19 & 19 & 17 & 9 & 10 & 14 & 5 & 2 & 22 & 15 & 19 & 9 & 8 & 10 \\
\hline 5 & 7 & 3 & 24 & 6 & 19 & 24 & 19 & 24 & 9 & 4 & 16 & 25 & 11 \\
\hline 8 & 8 & 7 & 12 & 21 & 15 & 13 & 16 & 14 & 6 & 7 & 6 & 10 & 12 \\
\hline 10 & 10 & 11 & 15 & 16 & 4 & 7 & 9 & 8 & 11 & 12 & 13 & 13 & 13 \\
\hline 24 & 21 & 24 & 2 & 25 & 23 & 23 & 20 & 23 & 23 & 18 & 8 & 5 & 14 \\
\hline 15 & 15 & 12 & 5 & 18 & 16 & 10 & 7 & 21 & 14 & 17 & 12 & 9 & 15 \\
\hline 9 & 9 & 14 & 17 & 8 & 11 & 20 & 23 & 9 & 20 & 13 & 10 & 17 & 16 \\
\hline 12 & 12 & 10 & 11 & 5 & 26 & 25 & 24 & 26 & 22 & 14 & 11 & 11 & 17 \\
\hline 23 & 23 & 18 & 21 & 15 & 13 & 11 & 5 & 10 & 13 & 16 & 23 & 22 & 18 \\
\hline 13 & 13 & 15 & 13 & 11 & 18 & 21 & 25 & 7 & 24 & 15 & 14 & 14 & 19 \\
\hline 17 & 17 & 20 & 18 & 13 & 25 & 26 & 26 & 4 & 26 & 23 & 15 & 18 & 20 \\
\hline 26 & 26 & 19 & 26 & 24 & 9 & 16 & 17 & 5 & 17 & 20 & 26 & 26 & 21 \\
\hline 21 & 25 & 22 & 25 & 22 & 8 & 12 & 10 & 15 & 18 & 26 & 24 & 23 & 22 \\
\hline 18 & 18 & 21 & 20 & 9 & 17 & 18 & 18 & 12 & 21 & 22 & 20 & 20 & 23 \\
\hline 20 & 20 & 23 & 14 & 14 & 24 & 19 & 13 & 25 & 16 & 21 & 19 & 16 & 24 \\
\hline 22 & 22 & 25 & 10 & 17 & 20 & 22 & 22 & 18 & 25 & 24 & 21 & 12 & 25 \\
\hline 25 & 24 & 26 & 22 & 23 & 10 & 14 & 15 & 19 & 19 & 25 & 22 & 21 & 26 \\
\hline
\end{tabular}


Figure 1

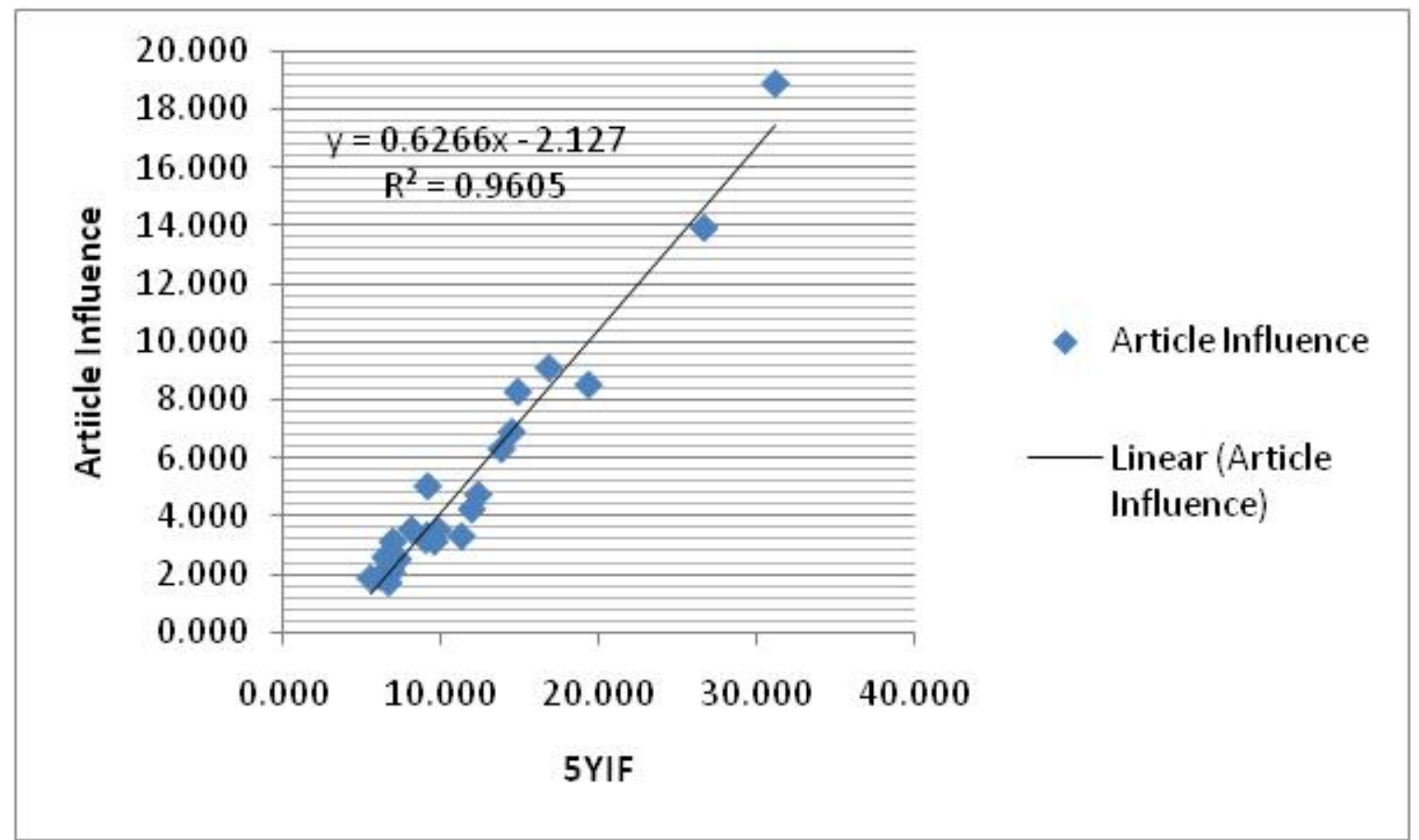

Note: Citations data for Neurosciences were downloaded from ISI on 12 June 2010 for 1988-2010.

Figure 2

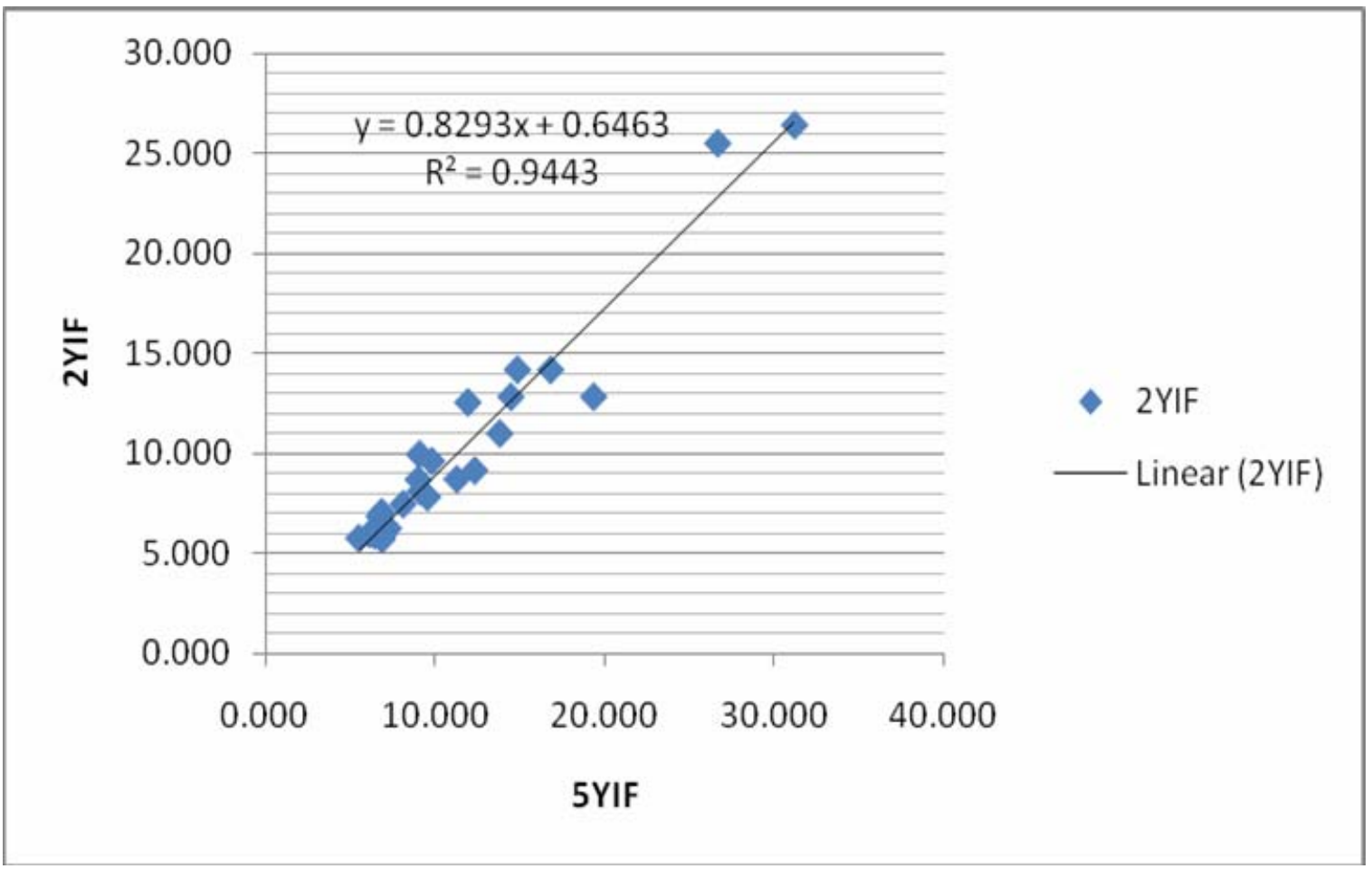

Note: Citations data for Neurosciences were downloaded from ISI on 12 June 2010 for 1988-2010. 


\section{Figure 3}

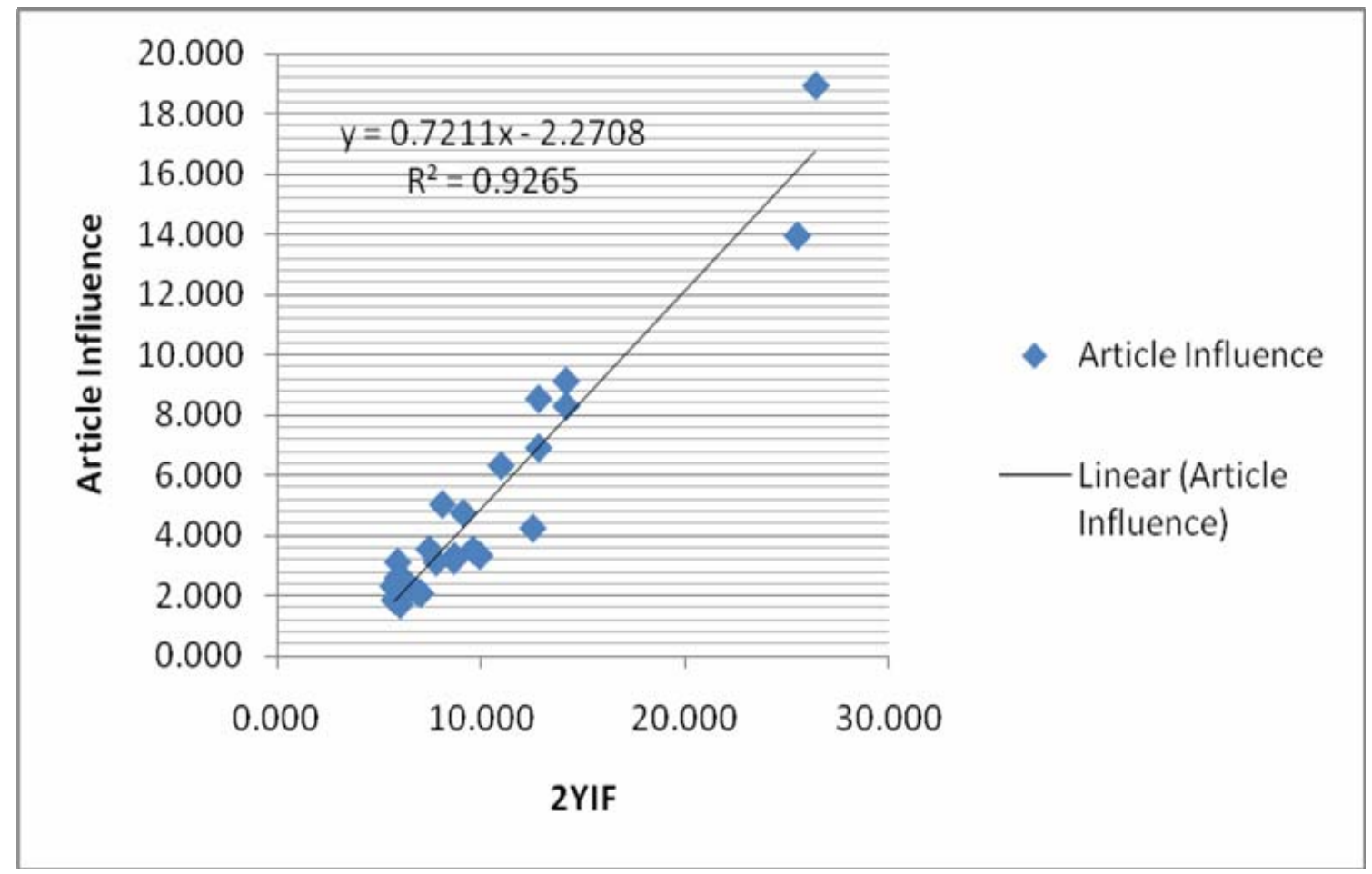

Note: Citations data for Neurosciences were downloaded from ISI on 12 June 2010 for 1988-2010.

\section{Figure 4}

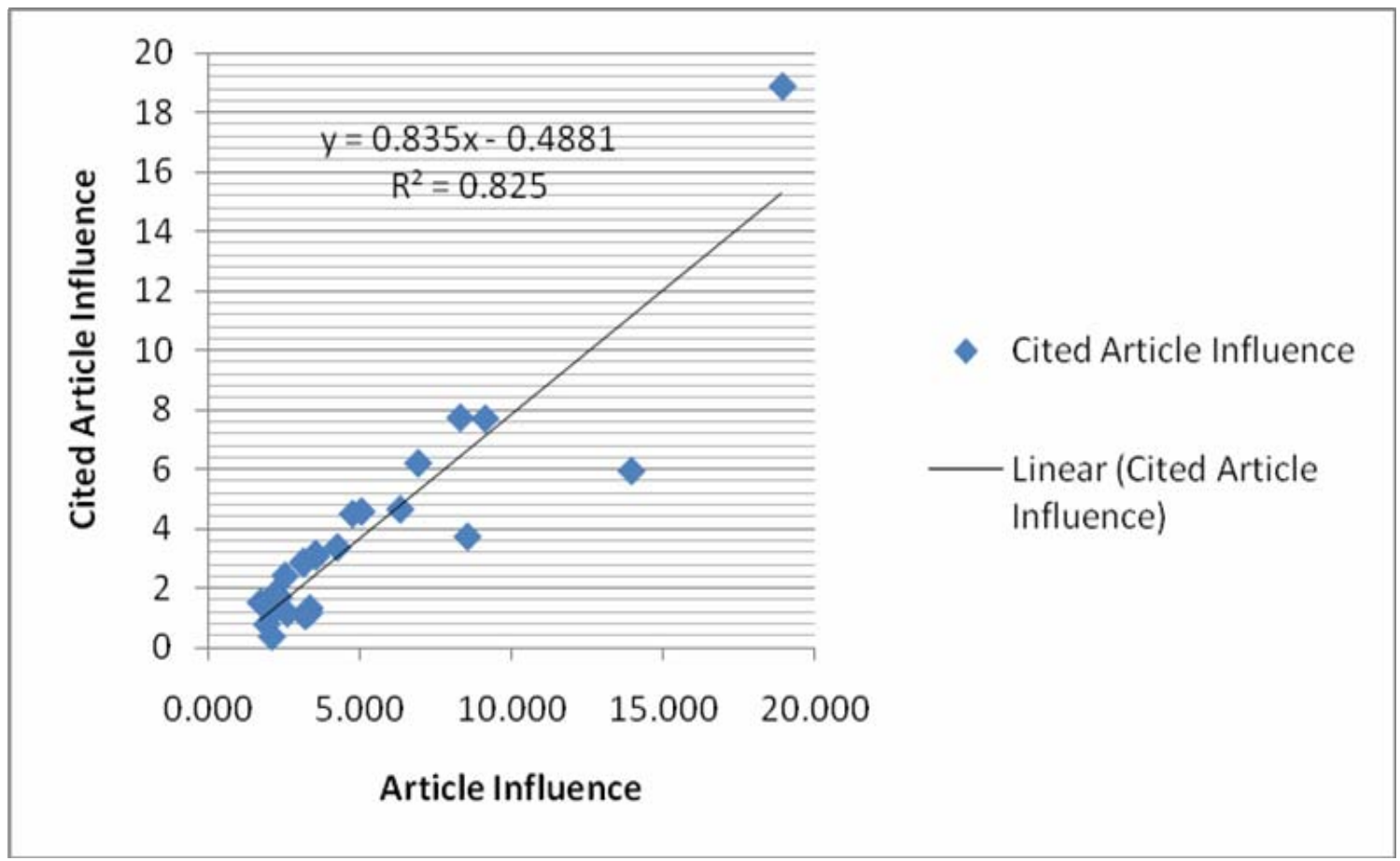

Note: Citations data for Neurosciences were downloaded from ISI on 12 June 2010 for 1988-2010. 